\title{
Sanal Flow Choking Leads to Hemorrhagic Stroke and Other Neurological Disorders in Earth and Human Spaceflight
}

V.R. Sanal Kumar, Shiv Kumar Choudhary, Pradeep Kumar Radhakrishnan, Suresh Menon, Vrishank Raghav, K.K.N.Namboodiri, Sapna E Sreedharan, Bharath R.S, Nichith C, Charlie Oommen, Vigneshwaran S, Ajith S, Arun Krishnan, Abhishesh Pal, Tharikaa R, Gayathri V.P, Abhirami Rajesh Group Namer Indo-US Investigators on Asymptomatic Stroke and Acute MI

\section{BACKGROUND}

Evidences are escalating on the diverse neurological disorders associated with COVID-19 pandemic due to the nanoscale Sanal flow choking (PMC7267099). The Sanal flow choking occurs at relatively high and low blood viscosity. Sanal flow choking leads to aneurysm, hemorrhagic-stroke and other neurological-disorders if the vessel geometry is having divergence, bifurcation, stenosis and/or occlusion regions (PMC7933821). Nanoscale Sanal flow choking is more susceptible at microgravity condition due to altered variations of blood viscosity, turbulence and the blood pressure ratio (BPR). Astronauts/Cosmonauts experienced neurological disorders during human spaceflight and thereafter.

V.R.S.Kumar et al. [1-7] reported that the asymptomatic episodes in the cardiovascular system are due to the internal flow choking (Biofluid/ Sanal flow choking (Fig.1)) at a critical blood pressure ratio (BPR), which is regulated by the biofluid/blood heat capacity ratio (BHCR).

\section{OBJECTIVE}

To establish the proof of the concept of the nanoscale Sanal flow choking in the cardiovascular system (CVS) causing asymptomatic-stroke and other neurological disorders on Earth and Human Spaceflight.

\section{METHODS}

Closed-form analytical, in vitro and in silico studies have been invoked herein for establishing the proof of the concept of nanoscale Sanal flow choking. Using in vitro methodology $\mathrm{BHCR}$ of various healthy subjects are estimated [6].

\section{DISCLOSURES}

Presenter: Dr. V. R. SANAL KUMAR Company Name: Indian Space Research Organization (SC CA No.6301/2013); India; Disclosure: None Prof. Shiv Kumar Choudhary All India Institute of Medical Sciences, New Delhi, India; Disclosure: None

Prof. Pradeep Kumar Radhakrishnan GITAM University, Visakhapatnam- 530 045, AP, India;

Disclosure: None

Prof. Suresh Menon

Georgia Tech, Atlanta, GA 30332-0150, U S A

Disclosure: None

Prof. Vrishank Raghav,

Auburn University, Auburn, AL 36849-5338, U.S.A:

Disclosure: None

Prof. K.K.Narayanan Namboodiri

Sree Chitra Tirunal Institute for Medical Sciences and

Tree Chitra Tirunal Institute for Medical Scic

Prof. Sapna E Sreedharan

Sree Chitra Tirunal Institute for Medical Sciences and

Technology, Kerala, India; Disclosure: None

Prof. Charlie Oommen, Bharath R.S, \& Nichith C.

Prof. Chatic O Osimen, Bharath R.S, \& Nichich

Disclosure: None

Vigneshwaran Sankar

Indian Institute of Technology, Kanpur, India;

Disclosure: None

A jith Sukumaran

Kumaraguru College of Technology, Coimbatore, India

Kumaraguru Colteg

Dr. Arun Krishnan

Dartmouth-Hitchcock Medical Center, Lebanon, NH 03756

U.S.A.; Disclosure: None

Dr. A bhishesh Pal.

The Norwegian U

Disclosure: None

Dr. Tharikaa Ramesh kumar

Auburn University, Auburn, AL 36849-5338, U.S.A;

Disclosure: None

Ms. Gayathri Vimal Raj Panicker

NMC Royal Hospital, Abudhabi; Disclosure: None

Ms. Abhirami Rajesh, Amrita Institute of Medical

Sciences, Kochi, Kerala, India, Disclosure: None

\section{R=SULTS}

The closed-form analytical models reveal that the relatively high and low blood viscosity are risk factors of Sanal flow choking. In vitro study shows that $\mathrm{N}_{2}, \mathrm{O}_{2}$, and $\mathrm{CO}_{2}$ gases are predominant in fresh-blood samples of the healthy human-being and Guineapig (Fig.2) at a temperature range of 37-40 $\mathrm{C}$ (98.6-1040 F), which increases the risk of Sanal flow choking. BHCR is found relatively high for healthy subjects with high BPR [6]. It is evident from our in silico results (Fig.3) that the Sanal flow choking leads to the shock-wave generation and transient pressure-overshoot in any artery with the divergence/ bifurcation regions.

\section{CONCLUSIONS}

As the pressure of the nanoscale biofluid / non-continuum-flows rises, fluid viscosity increases and averagemean-free-path diminishes and thus, the Knudsen number lowers heading to a zero-slip wall-boundary condition with the compressible flow regime, which increases the risk of Sanal flow choking and the shock wave generation causing asymptomatic cardiovascular disease. Microgravity environment decreases plasma volume and increases the hematocrit compared with the situation on the Earth surface, which increases the relative viscosity of blood causing an early Sanal flow choking. Herein, we established that the disproportionate blood-thinning treatment increases the risk of the nanoscale Sanal flow choking due to the enhanced boundary-layer-blockage factor. The risk could be diminished by concurrently reducing the viscosity of biofluid/ blood and flow-turbulence by increasing the thermal-tolerance-level in terms of BHCR and/or by decreasing the BPR through new drug discovery or using companion medicine with the traditional blood thinners or other health care management. We recommend all astronauts/cosmonauts should wear ambulatory blood pressure and thermal level monitoring devices similar to a wristwatch throughout the space travel for the diagnosis, prognosis and prevention of internal flow choking leading to asymptomatic cardiovascular disease including neurological disorders.

\section{INTRODUGTION}

The large vessel stroke and the increased incidence of cryptogenic stroke in multiple regions have been reported in Covid-19 patients [8-11]. V.R.S.Kumar et al. [1-7] reported that hemorrhagic-strokes in multiple regions of various vessels causing neurological disorders are due to internal flow choking (See Fig.1). This is particularly true for astronauts/ cosmonauts who experienced neurological disorders due to nanoscale Sanal flow choking during human spaceflight and thereafter [6].

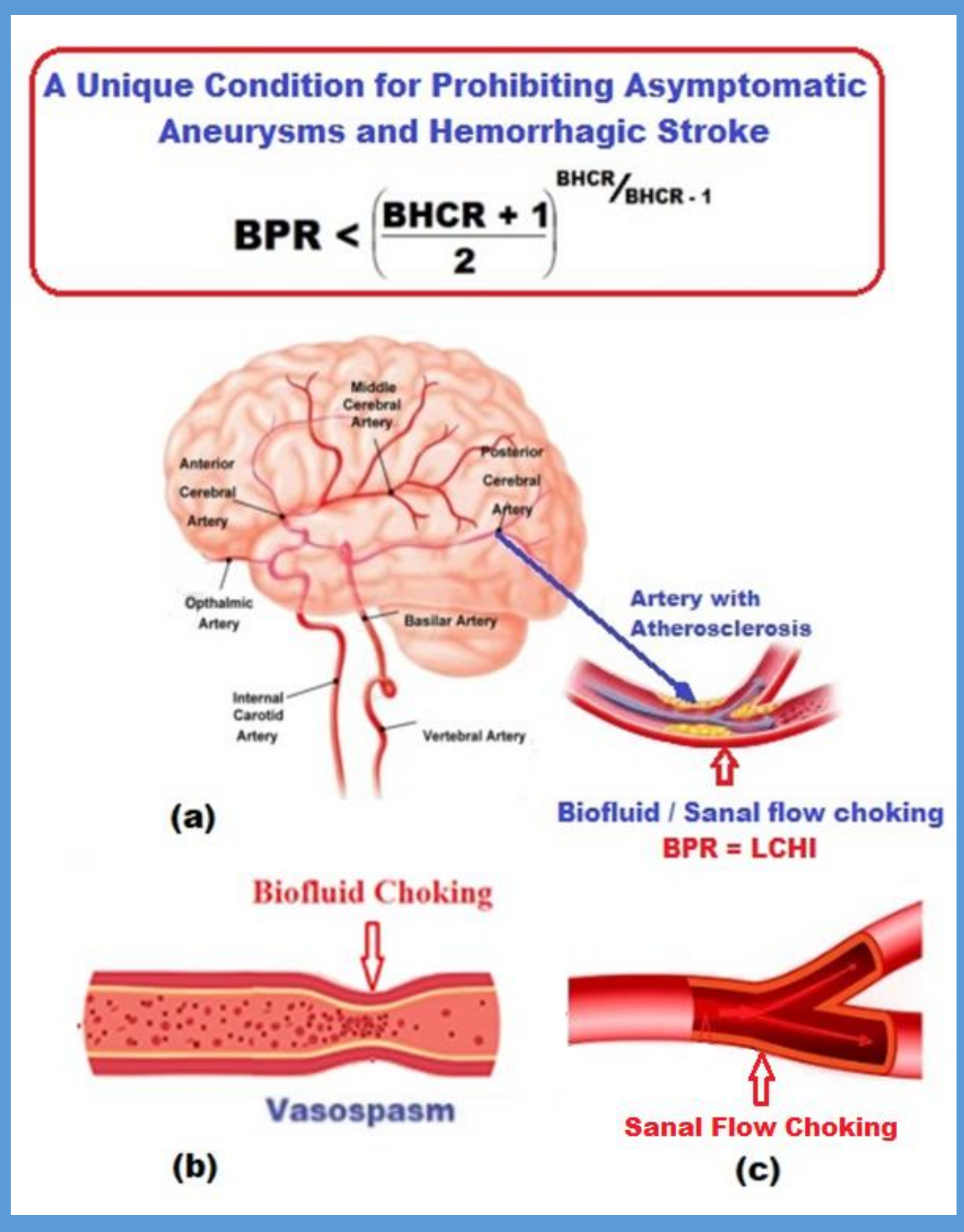

Figure 1(a-c). Demonstration of different physical situations of internal flow choking (Biofluid / Sanal flow choking) at a critical blood-pressure-ratio $(B P R=$ SBP/DBP $)$.

Hemorrhagic-stroke (PMC7163302) and other neurological disorders could occur in hypertension and hypotension patients because the controlling parameter of these asymptomatic episodes are the critical BPR [4]. The critical BPR (SBP/DBP) for internal flow choking is regulated by BHCR.

\section{ANALYTIGAL RESULTS}

Flow choking is more prone during the winter season than the summer season due to the higher blood viscosity at the relatively low blood temperature. To avoid internal flow choking the BPR must always be lower than the lower critical hemorrhage index (LCHI).

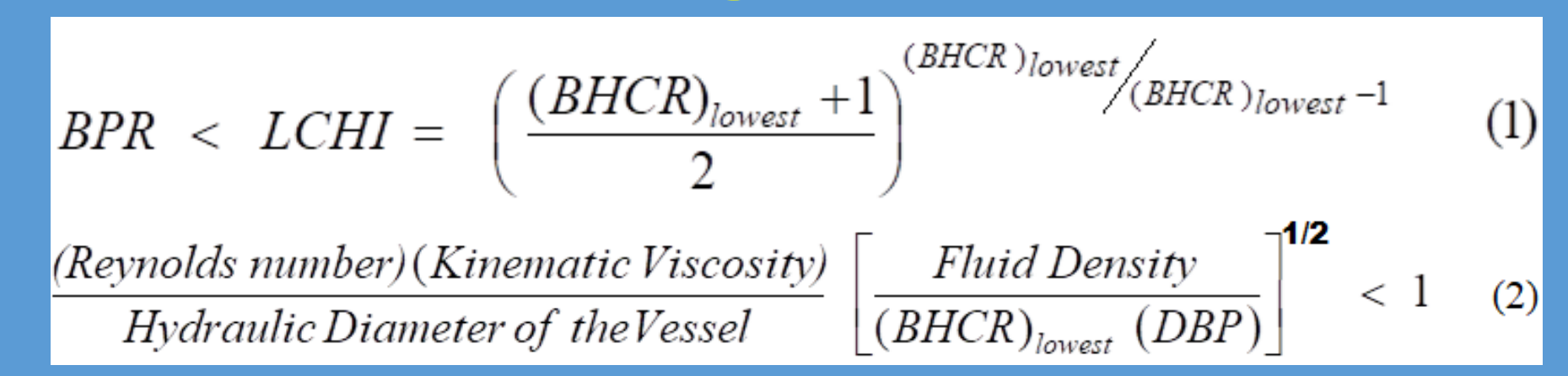

Equations 1 \& 2 are complementing conditions set for prohibiting the internal flow choking in the CVS.

\section{IN Vhro RESUL'S}

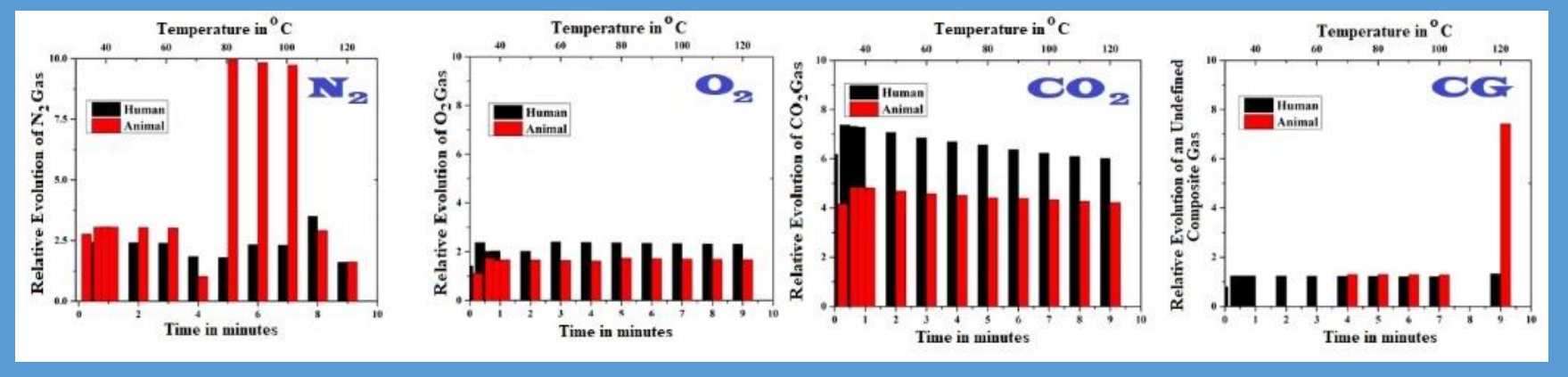
Figure 2. Mass spectrum of $\mathrm{N}_{2}, \mathrm{O}_{2}, \mathrm{CO}_{2}$ and an unknown composite gas (CG)

IN SILICO RESUL's

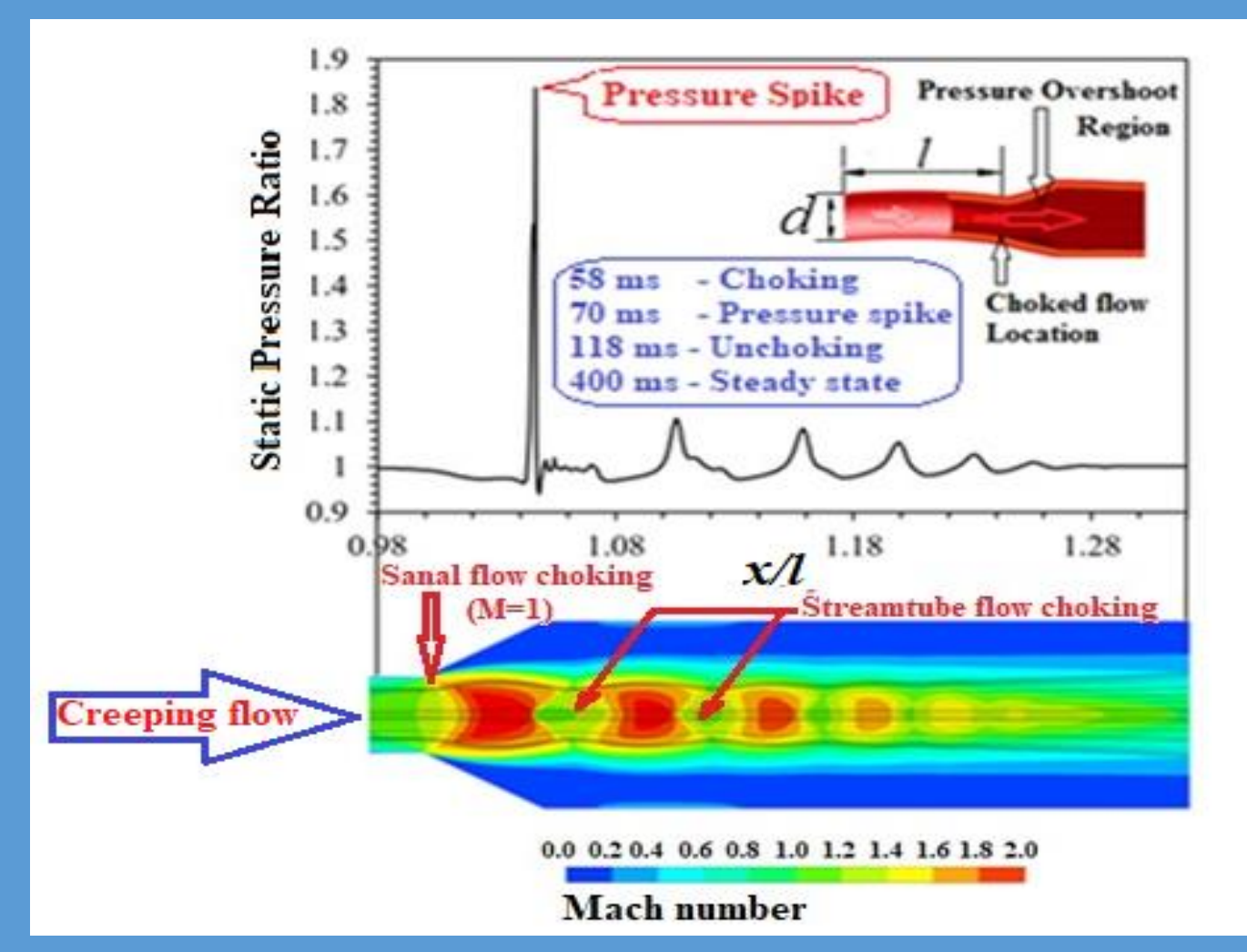

Figure 3. Single phase in silico result: Demonstration of Sanal flow choking and trancient pressure-overshoot.

We envisaged that development of a multi-phase, multispecies, viscoelastic fluid-structural interactive in silico model capturing the memory effect (stroke history) is a meaningful objective for diagnosis, prognosis and prevention of asymptomatic episodes in CVS.

RAFERENGES

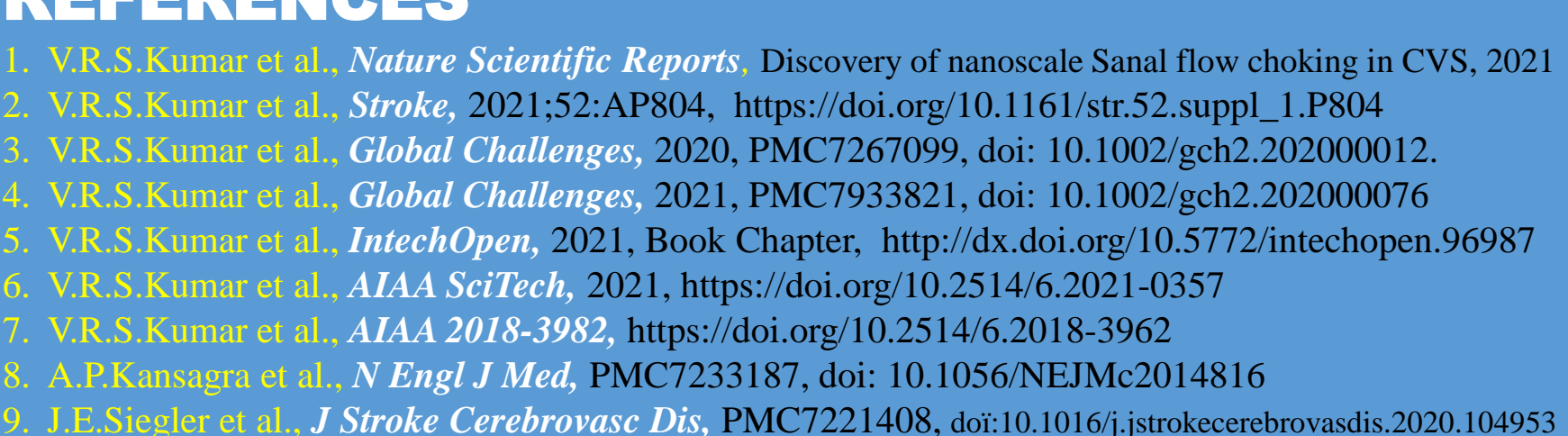

